Revue internationale P.M.E.

\title{
L'apport d'internet aux petites entreprises (PE) touristiques dans la construction de leur positionnement stratégique : le cas des hébergeurs
}

\section{The contribution of the internet to tourism small enterprises (SEs) in developing their strategic positioning: the case of hosting service providers \\ La contribución de internet para las empresas pequeñas (PE) turísticasen el desarrollo de su posicionamiento estratégico: el caso de los anfitriones}

\section{Véronique Favre-Bonté et Sébastien Tran}

Volume 26, numéro 1, 2013

URI : https://id.erudit.org/iderudit/1024270ar

DOI : https://doi.org/10.7202/1024270ar

\section{Aller au sommaire du numéro}

Éditeur(s)

Editions EMS - In Quarto SARL

ISSN

0776-5436 (imprimé)

1918-9699 (numérique)

Découvrir la revue

Citer cet article

Favre-Bonté, V. \& Tran, S. (2013). L’apport d'internet aux petites entreprises (PE) touristiques dans la construction de leur positionnement stratégique : le cas des hébergeurs. Revue internationale P.M.E., 26(1), 45-64.

https://doi.org/10.7202/1024270ar

\section{Résumé de l'article}

La diffusion des TIC chez les PE demeure faible (Eurostat 2010) et pourtant, parmi les applications IT, Internet est pour les PE une source d'opportunités de développement de leurs affaires (Scaglione, Schegg et Murphy, 2009). L’un des enjeux est donc de savoir si Internet peut être utilisé efficacement par ces petites structures et comment sa mise en oeuvre peut contribuer à améliorer leur positionnement stratégique. Nous avons étudié quatre cas d'entreprises dans l'hébergement touristique qui est un secteur très en avance dans l'utilisation des TIC en général et d'Internet en particulier (Kalika et Rival, 2009 ; Aldebert et Loufrani-Fedida, 2010). Notre recherche montre que les PE peuvent utiliser Internet comme un outil de différenciation, notamment sur les dimensions liées à la relation client (marketing, distribution). La réussite, quant à l'utilisation d'un site Internet, repose sur un choix très judicieux de la technologie par rapport au positionnement de l'entreprise. La mise en place d'une stratégie doit donc se faire à partir d'une véritable réflexion quant au métier de la PE, car cela conditionne les services et les fonctionnalités proposées à partir des technologies. Enfin, ce n'est pas tant le profil technophile qui importe que le profil innovant et preneur de risques du dirigeant et son aptitude à percevoir l'utilité des technologies. 


\title{
L'apport d'internet aux petites entreprises (PE) touristiques dans la construction de leur positionnement stratégique : le cas des hébergeurs
}

\begin{abstract}
Véronique FAVRE-BONTÉ
Véronique Favre-Bonté est Maître de conférences à l'Université de Savoie (membre de l'IREGE, Institut de recherche en gestion et économie) où elle enseigne la stratégie, l'intelligence économique et la théorie des organisations. Elle est responsable d'un master 2 Commerce Responsable à l'International et d'une licence professionnelle Affaires Internationales. Ses recherches et publications se focalisent sur les stratégies internationales et l'innovation dans les services.

Institut de recherche en gestion et économie Université de Savoie (IAE Savoie Mont-Blanc), laboratoire IREGE veronique.favre-bonte@univ-savoie.fr
\end{abstract}

\section{Sébastien TRAN}

Sébastien Tran est Doyen de la faculté de l'EM Normandie en France, enseignant-chercheur en stratégie et chercheur associé au laboratoire M-Lab de l'Université Paris Dauphine (DRM - UMR CNRS 7088). Ses recherches portent sur les TIC, les SI et le management de l'innovation. Il est l'auteur de plusieurs articles et communications dans les différentes communautés académiques. Il est également membre du CA de l'AIM depuis 2011.

EM Normandie, chercheur associé à M-Lab, Université Paris Dauphine s.tran@em-normandie.fr

\footnotetext{
RÉSUMÉ

La diffusion des TIC chez les PE demeure faible (Eurostat 2010) et pourtant, parmi les applications IT, Internet est pour les PE une source d'opportunités de développement de leurs affaires (Scaglione, Schegg et Murphy, 2009). L'un des enjeux est donc de savoir si Internet peut être utilisé efficacement par ces petites structures et comment sa mise en cuvre peut contribuer à améliorer leur positionnement stratégique. Nous avons étudié quatre cas d'entreprises dans l'hébergement touristique qui est un secteur très en avance dans l'utilisation des TIC en général et d'Internet en particulier (Kalika et Rival, 2009; Aldebert et Loufrani-Fedida, 2010). Notre recherche montre que les PE peuvent utiliser Internet comme un outil de différenciation, notamment sur les dimensions liées à la relation client (marketing, distribution). La réussite, quant à l'utilisation d'un site Internet, repose sur un choix très judicieux de la technologie par rapport au positionnement de l'entreprise. La mise en place d'une stratégie doit donc se faire à partir d'une véritable réflexion quant au métier de la PE, car cela conditionne les services et les fonctionnalités
} 
proposées à partir des technologies. Enfin, ce n’est pas tant le profil technophile qui importe que le profil innovant et preneur de risques du dirigeant et son aptitude à percevoir l'utilité des technologies.

MOTS CLÉS

Internet, TIC, PE, Tourisme.

\title{
The contribution of the internet to tourism small enterprises (SEs) in developing their strategic positioning: the case of hosting service providers
}

\begin{abstract}
The diffusion of ICT in small businesses remains low (Eurostat 2010) and yet among IT applications, the Internet offers opportunities for such businesses to develop their business activity (Scaglione, Schegg et Murphy, 2009). One issue is whether the Internet can be used effectively by these small structures and how the use of this technology can improve the strategic position of small businesses. Our qualitative methodology is based on a study of four companies working in the area of tourist accommodation, a sector which is very advanced in the use of ICT in general and the Internet in particular (Kalika and Rival, 2009; Aldebert et Loufrani-Fedida, 2010). Our research shows that small businesses can use the Internet as a tool for differentiation, notably in areas related to customer relations (marketing, distribution). The successful use of a website depends on a very wise choice of technology linked to the marketing position of the company. The implementation of a strategy must be the result of a deep reflection on the activity of the business because it determines the services and features offered by technology. Finally, it is not so much having a technophile profile that is important, but rather the ability of leaders to perceive the usefulness of technology through their innovative and risk-taking profile.
\end{abstract}

KEY WORDS

Internet, ICT (Information and Communication Technology), SB (Small Business),

Tourism.

\section{La contribución de internet para las empresas pequeñas (PE) turisticasen el desarrollo de su posicionamiento estratégico: el caso de los anfitriones}

\begin{abstract}
RESUMEN
En las Pequeñas Empresas (PE), la difusión de las TIC (Tecnologías de Información y Comunicación) sigue débil; sin embargo, entre las aplicaciones IT, Internet es una fuente de oportunidades de desarrollo para sus negocios (Scaglione et al. 2009). Lo que está en juego, es saber si puede utilizarse eficazmente el Internet para estas pequeñas estructuras y cómo su implementación puede contribuir al mejoramiento de su posición estratégica. Hemos estudiado cuatro casos de empresas dedicadas al alojamiento turístico, sector que se encuentra muy avanzado en el uso de las TIC en general y particularmente de Internet (Kalika y Rival, 2009 ; Aldebert y Loufrani-Fedida, 2010). Nuestra investigación revela que las PE pueden usar Internet como una herramienta de diferenciación especialmente relacionada con la atención al cliente (marketing, distribución). El éxito, en cuanto al uso de un sitio web, radica en la elección sensata de la tecnología
\end{abstract}


según la posición de la empresa. Poner en práctica una estrategia, debe entonces hacerse a partir de una auténtica reflexión en cuanto a la función de la PE ya que esto condiciona los servicios y las funcionalidades propuestas a partir de las tecnologías. Por último, lo que importa no es tanto el perfil técnico sino más bien su perfil innovador y arriesgado y su capacidad de entender la utilidad de las tecnologías.

\section{Palabras Clave}

Internet. TIC (Technologias de Información y Comunicación), PE (Pequeñas Empresas), Turismol.

\section{INTRODUCTION}

Les $\mathrm{PME}^{1}$ représentent une part significative des entreprises dans les pays industrialisés. À titre d'exemple, elles représentaient près de $97 \%$ des entreprises en France en 2007 (INSEE). De nombreux travaux ont ainsi porté leur attention à leurs problématiques, que ce soit sur leur modèle économique, leur gestion ou leur stratégie (Julien et Marchesnay, 1988 ; Julien, 1998). Néanmoins, il existe peu de travaux sur les petites entreprises (PE). Ces dernières, dont l'effectif salarié est inférieur à 50 personnes dans la plupart des définitions officielles, représentaient en 2003 près de $92 \%$ des entreprises européennes selon l'observatoire européen des PME (Harindranath, Dyerson et Barnes, 2008)2. Par ailleurs, le secteur du tourisme représente un poids économique majeur en France ${ }^{3}$ avec un nombre important de PME et de TPE puisqu'on en comptabilisait 235000 en 2009, en France ${ }^{4}$. Ce secteur est également très en avance dans l'utilisation des TIC où les informations sont au cœur des prestations (Kalika et Rival, 2009 ; Aldebert et Loufrani-Fedida, 2010). En 2010, le tourisme en ligne a pesé plus de 8 milliards d'euros (hors voyages d'affaires), en croissance de $19 \%$ par rapport à 2009. Après une croissance de $6 \%$ en 2009 , le tourisme profite largement de la reprise de la croissance du marché dans son ensemble. Toujours selon CCM Benchmark, le tourisme en ligne représente ainsi, en 2010, 46,1\% de l'ensemble du commerce en ligne B to $\mathrm{C}$ en France, notamment via la multiplication des offres en ligne issues des sites Internet.

1 Les PME au sens de l'INSEE sont les entreprises de moins de 250 salariés et dont le chiffre d'affaires n'excède pas 50 millions d'euros ou le total de bilan n'excède pas 43 millions d'euros (INSEE).

2 À titre de point de repère et dans un focus encore plus restreint que les PE, la France comptait 2,3 millions de TPE ( 0 à 19 salariés) en 2006 qui représentaient $30 \%$ de lemploi salarié (rapport Mission TIC et PME du Ministère des PME, du Commerce, de l'Artisanat et des Professions libérales).

3 Selon le site Internet de l'OMT, les recettes en 2008 du tourisme en France ont représenté 55,6 milliards de \$, positionnant ainsi le pays en troisième position dans le monde.

4 Le poids économique et social du tourisme, Conseil National du Tourisme, Minefi, 2010. Il est à noter que ce nombre augmente au rythme moyen de 1,6\% par an depuis 2000. 
Ces éléments suggèrent une utilisation très orientée sur les dimensions commerciale et marketing des TIC, hypothèse confirmée dans quelques travaux. Létude de Bonn, Furr et Susskind (1998) montre que le marketing via Internet est approprié aux produits et services touristiques. Or, en France, la diffusion au sein des PE demeure encore faible : seules $12 \%$ ont un site Internet permettant d'effectuer des transactions en ligne (Eurostat 2010). Même si les petites structures sont conscientes des principaux bénéfices offerts par les TIC, $46 \%$ des PME en France restent néanmoins sceptiques quant à leur rôle dans le développement de leur chiffre d'affaires (France Numérique 2012, p. 56). La préoccupation actuelle des institutions et gouvernements réside donc dans l'utilisation et l'apport des technologies au développement des entreprises ${ }^{5}$. Ces efforts reposent aussi sur le fait que les TIC $^{6}$ dans les entreprises sont considérées comme un des facteurs de renouvellement des conditions de compétitivité, notamment en permettant un relâchement des contraintes spécifiques à la PME (Sullivan, 1985 ; Raymond, Bergeron et Blili, 2005 ; Amabille et Gadille, 2006 ; Qian et al. 2006 ; Matthews, 2007 ; Bellaaj, 2009). On peut dès lors s'interroger si certaines TIC, tels que les sites Internet, ne contribuent pas à faire évoluer le positionnement stratégique des petites structures, notamment les PE.

Compte tenu de l'hétérogénéité des activités dans le tourisme, nous avons centré notre attention sur l'activité d'hébergement au sens large (camping, hôtellerie, thalassothérapie...) qui représente plus de $20 \%$ des entreprises du secteur en France en 2007 (INSEE, EAE services 2007) et qui constitue souvent le cœur de l'activité touristique (c'est la seconde activité du secteur en matière de chiffre d'affaires après la restauration). Notre problématique sera donc la suivante : sous quelles conditions la mise en place d'un site Internet peut-elle modifier le positionnement stratégique de petites structures d'hébergement dans le secteur du tourisme?

Cet article poursuit un double objectif. Il s'agit tout d'abord de montrer que malgré leur petite taille, des entreprises sont capables d'utiliser Internet de façon « experte " pour se développer sur le plan marketing et commercial. Il s’agit également d’expliquer quà partir d'un certain stade et de certaines conditions, Internet peut aussi devenir un levier de positionnement stratégique. Dans une première partie, nous ferons un état de l'art des rôles que peut jouer Internet dans le développement des petites structures. Dans une deuxième partie, après avoir exposé nos choix méthodologiques et les cas étudiés, nous discuterons des résultats issus de notre terrain afin non seulement de signaler l'impact de l'utilisation d'un site Internet pour les hébergeurs, mais aussi d'en tirer un certain nombre d'implications pour les managers.

5 Plusieurs initiatives gouvernementales (le passeport pour l'économie numérique en France, The European e-Business Support Network for SMEs au niveau européen, etc.) ont été développées pour inciter les PME, souvent en retard par rapport aux grandes entreprises, à la mise en place d'une politique en matière de TIC.

6 Derrière cette abréviation se cachent une multitude d'applications (courrier électronique, Intranet, Extranet, Groupware...) dont le déploiement se révèle être particulièrement hétérogène, même au sein d'un secteur. Afin d'englober cette diversité, nous définirons les TIC comme des techniques permettant de saisir, traiter, stocker et communiquer l'information (Reix, 2002). 


\section{QUELS RÔLES JOUENT LES TIC ET INTERNET EN PARTICULIER DANS LE DÉVELOPPEMENT DES PETITES STRUCTURES ?}

Les petites structures, notamment les PE, malgré les contraintes inhérentes à leur petite taille, peuvent utiliser les TIC afin de maintenir ou de développer leur avantage concurrentiel dans un environnement économique de plus en plus difficile (renforcement de la concurrence, internationalisation, etc.). Or les TIC seules n’ont pas de caractère à créer de la valeur, c'est lorsqu'elles sont combinées à d'autres ressources ou dans un contexte particulier qu'elles sont créatrices de valeur. Nous allons voir comment et sous quelles conditions les TIC et Internet en particulier peuvent jouer un rôle au niveau marketing (1.1), mais également un rôle stratégique (1.2) dans les petites structures. Nous aborderons aussi le dirigeant qui, selon ses caractéristiques et son implication, va avoir un effet « amplificateur » sur cette création de valeur et être une des conditions de lévolution de la petite structure (1.3).

\subsection{Internet comme levier d'action marketing et commercial des petites structures}

D’une façon générale, les recherches montrent que l'utilisation des TIC au sein des petites structures est surtout focalisée sur des utilisations opérationnelles et très peu sur des orientations stratégiques (Dyerson, Harindranath et Barnes, 2009). Selon Lee et Morrison (2010), il y a plusieurs avantages majeurs qui résultent d'une présence sur le Web : réduction des coûts, croissance des revenus, marketing ciblé, amélioration de la satisfaction client, amélioration de la qualité, réponse aux besoins clients. Pour autant, il est difficile d'évaluer le retour précis de l'utilisation d'Internet pour les entreprises. Le prisme des recherches menées se fait donc le plus souvent sur les bénéfices perçus, notamment sur les dimensions commerciales et marketing, qui demeurent une motivation prépondérante chez les dirigeants de petites structures (Mehrtens, Cragg et Mills, 2001 ; Dyerson et al., 2009).

L'un des points les plus importants concerne le développement du commerce en ligne pour les entreprises (Raymond et al., 2005 ; Bellaaj, 2009 ; Dyerson et al., 2009), avec toutefois très peu d'entreprises allant jusqu'à la commande et au règlement en ligne (Lucchetti et Sterlacchini, 2004). Bien que cela ne reste qu'un canal marginal, les petites structures utilisent aussi les TIC pour mettre en place une tarification sophistiquée, en temps réel, permettant ainsi l'utilisation des techniques de yield management et de tarification dynamique (Gensollen, 2001 ; Aldebert et Loufrani-Fedida, 2010). Toutefois, seule une partie du processus de vente est souvent dématérialisée, notamment en amont de la commande (communication, catalogues, etc.). Ceci s'explique en partie par le manque de ressources financières et humaines. L'investissement dans des solutions logicielles appropriées a un coût non négligeable pour une petite structure. À l'équipement de base, s'ajoutent également les coûts de formation et de maintenance (McDonagh et Prothero, 2000 ; Montazemi, 2006 ; Matthews, 2007 ; Dyerson et al., 2009).

Dans la réflexion sur la dimension commerciale, les TIC, et Internet en particulier, sont également un moyen d'améliorer la relation client en maintenant un lien plus régulier et interactif avec certains segments de consommateurs et en diffusant des informations ciblées 
(Levenburg, 2005 ; Qureshil, Kamal et Wolcott, 2009). La visibilité de l'entreprise est aussi un des aspects soulignés dans quelques travaux, sans que ne soit visé directement un objectif commercial, mais plutôt dans le souci d'accroître la notoriété (Mehrtens et al., 2001 ; Lucchetti et Sterlacchini, 2004). Le développement des sites Internet, associé à la réduction des coûts de leur mise en place, constitue souvent une opportunité de créer une vitrine pour l'entreprise dont le rayonnement géographique et les moyens sont limités. Enfin, l'amélioration de la communication au sens large demeure aussi une des motivations principales.

Dans les petites structures, la notion d'avantage concurrentiel se résume souvent à une dimension commerciale et une dimension plus centrée sur le client (Amabille et Gadille, 2003; 2006), néanmoins, certaines engagent une vraie réflexion stratégique grâce à la mise en place de leur site Internet, notamment lorsquelles ont atteint un stade de maîtrise et de maturité en matière de fonctionnalités et de services (la conclusion de la transaction et le paiement en ligne étant une des phases les plus avancées). La petite structure vise, via Internet, à rechercher une différenciation sur le marché. Cette différenciation consiste à jouer non pas sur une réduction des prix, mais sur un différentiel de valeur perçu par le client (Aldebert, 2008).

\subsection{Internet peut constituer également un outil de positionnement stratégique pour les petites structures}

Nous venons de voir que les TIC, et notamment Internet, peuvent permettre aux petites structures d'enrichir leur stratégie commerciale et marketing (tarification dynamique, visibilité accrue auprès de nouveaux clients, etc). Or, plus les petites structures utilisent Internet comme canal de communication et de relation avec leurs clients, plus la perception de ces derniers se modifie et s'améliore par rapport à leurs concurrents directs et aux concurrents de taille plus importante (Morrison, Taylor, Morrison et Morrison, 1999). Cela est surtout valable pour les petites structures qui sont à un stade avancé de l'utilisation d'Internet.

Internet n'apparait donc plus seulement comme un outil marketing, mais comme un déclencheur d'une dynamique qui peut initier des réflexions stratégiques (Monnoyer-Longé et Madrid, 2007). Dans leur recherche, les auteurs précisent que le site web apparaît comme porteur de ressources organisationnelles et stratégiques qui ne se révèlent que dans le cadre d'une congruence entre gestion des TIC et choix stratégiques, congruence qui se met en place pour des entreprises " en avance ». Scaglione et al. (2009) montrent aussi que les taux de croissance des revenus des hôtels qui ont leur propre nom de domaine sont supérieurs à ceux qui ont une présence sur Internet via des portails régionaux : Internet devient alors un levier de positionnement stratégique pour ces entreprises qui sont à un stade avancé d'utilisation. Il convient donc d'identifier ces entreprises. Pour ce faire, deux typologies se révèlent pertinentes et relativement convergentes quant à leurs conclusions : la typologie de Daniel, Wilson et Myers (2002) et celle d'Aldebert (2008).

Dans la typologie de Daniel et al. (2002), les auteurs identifient quatre groupes correspondant à quatre stades d'utilisation des TIC : les entreprises développant leurs premiers services de commerce électronique (stade 1), celles qui utilisent le courrier électronique pour communiquer avec les clients, les fournisseurs et les employés (stade 2), celles qui ont mis en place des sites web basés sur les informations et développent des moyens de commande en ligne (stade 3). Enfin, les 
«adopters les plus avancés » (stade 4) ont mis en place la commande en ligne et développent des moyens de paiement en ligne (dernière colonne du tableau de l'annexe 1). Ce stade 4 correspond aux entreprises pionnières (de la typologie d'Aldebert, 2008), elles revêtent des caractéristiques bien particulières : tout d'abord, les efforts commerciaux ne sont pas vus comme de simples activités de distribution, mais comme une occasion de créer de la valeur ajoutée. Ensuite, stratégiquement, leurs dirigeants visent des segments de marché par différenciation ou spécialisation et ils pratiquent des innovations, aussi bien dans les produits, les services, les processus, les types de clientèle, que dans l'organisation interne de l'entreprise. Cela permet aux entreprises de ne pas être seulement connues, mais reconnues. Enfin, ces entreprises ont un comportement d'anticipation des marchés et donc un comportement proactif.

Les petites structures peuvent donc venir concurrencer des acteurs de taille plus importante avec un positionnement clair et différenciant, avec beaucoup moins de moyens. Elles sont alors à même de modifier la perception des consommateurs avec leur site Internet qui devient la vitrine de leur entreprise et donc, un élément important de positionnement (même si un site peut coûter cher, sa valeur ajoutée peut s'avérer très importante) (Morrison et al., 1999). De façon générale, les TIC n’ont pas d’effet direct sur le positionnement de l'entreprise sur son marché ou encore sur les performances de l'entreprise, mais c'est au travers de processus organisationnels et de conditions de diffusion simples qu'elles auront un impact sur l'entreprise (au niveau stratégique ou financier). De manière générale et quelle que soit la taille de l'entreprise, elles impliquent alors une modification de l'organisation et un alignement stratégique des ressources, des technologies et de la stratégie (Henderson et Venkatraman, 1993). Ainsi, selon Bergeron, Raymond et Croteau (2001), les stratégies d'investissement en TIC doivent être co-alignées avec les comportements stratégiques généraux de l'entreprise pour en affecter positivement la performance. Il est donc important de s'intéresser aussi au dirigeant qui semble jouer un rôle primordial dans cet alignement stratégique via la diffusion des technologies et l'utilisation d'Internet en particulier dans la petite entité.

\subsection{Le rôle du dirigeant dans l'utilisation des TIC dans les petites entités}

Il existe diverses caractéristiques permettant de dresser un profil organisationnel de la petite structure (Marchesnay, 2003 ; Julien, 1998) : une forte centralisation de la gestion et des prises de décision, une stratégie implicite de court terme et une faible décomposition des tâches privilégiant la polyvalence à la spécialisation. Ces caractéristiques se retrouvent dans les recherches sur les entreprises de petite taille, car elles mettent en avant non seulement l'importance du propriétaire-dirigeant dans les décisions (Julien et Marchesnay, 1988), notamment en ce qui concerne les choix en matière de technologies de l'information (Bergeron et al., 2001 ; Cragg, King et Hussinc, 2002 ; Monnoyer-Longé, 2003), mais également l'importance de la perception du dirigeant dans ses choix (Bergeron et al., 2001) et de son intérêt pour les technologies (Aldebert et Gueguen, 2009).

Selon la littérature (Mehrtens et al., 2001 ; Beckinsale, Levy et Powell, 2006), le premier élément explicatif de l'utilisation des TIC concerne les caractéristiques du dirigeant et son profil. Ainsi, les dirigeants ayant certaines caractéristiques (jeune âge, sensibilité aux technologies, niveau de compétences, etc.) seront plus enclins à utiliser les TIC. Cela rejoint également le niveau de bénéfices perçus des TIC par les décideurs (Pollard et Hayne, 1998 ; McDonagh 
et Prothero, 2000), notamment si la manière de conduire les affaires et le développement de l'entreprise est jugée satisfaisante en interne. À l'inverse, les barrières de nature psychologique et la peur du changement peuvent constituer des éléments explicatifs du faible niveau d'utilisation des TIC dans les PME (Acar, Kacak, Sey et Arditi, 2005) et a fortiori dans les petites structures. Enfin, ce manque de compétences et d'expertise techniques, mais également managériales (Caldeira et Ward, 2002 ; Matthews, 2007 ; Dyerson et al., 2009 ; Qureshil et al., 2009) peut être accentué par le risque de dépendance aux éditeurs de solutions logicielles qui sont dans une position privilégiée grâce à leur expertise technique.

Notons toutefois que les compétences de mise en œuvre des TIC peuvent être détenues par une autre personne que le dirigeant. D’une façon générale, la présence d'un utilisateur " expert ", que ce soit le dirigeant ou un autre membre de l'entreprise, est donc de nature à réduire la barrière de l'expertise technologique et à faciliter l'adoption des outils. La présence de collaborateurs compétents en matière de technologies est donc souvent une des conditions pour leur adoption au sein des petites structures. On retrouve également dans les facteurs explicatifs, le rôle joué par les parties prenantes proches de l'entreprise, à savoir les clients et les fournisseurs, ainsi que les autres prescripteurs (collectivités territoriales, associations professionnelles...) (Burgess, 2002). Les médias et prestataires de solutions logicielles sont également des acteurs qui peuvent conduire les dirigeants à séquiper en TIC. Naturellement, le poids de chacun de ces acteurs peut varier en fonction du secteur d'activité de l'entreprise.

En conclusion de cette première partie, il apparaît que les applications Internet sont orientées vers la gestion de la relation client, à la fois en amont (recherche de nouveaux clients, actualisation de l'offre, etc.), mais également en aval (échange d'informations, marketing relationnel, etc.). Il apparaît également qu'Internet peut constituer un outil de positionnement stratégique, notamment lorsque le dirigeant s'implique et arrive à intégrer l'outil dans sa vision. Ce dernier, même s'il ne possède pas les compétences techniques et managériales propres aux TIC, peut, grâce à sa perception des bénéfices et à son réseau, élaborer une stratégie marketing et commerciale pour développer son activité sur Internet. Cette présence sur le web peut, à partir d'un certain niveau, conduire à une évolution du positionnement stratégique de son entreprise.

\section{2. ÉTUDE EMPIRIQUE DE QUATRE PETITES STRUCTURES DANS LE DOMAINE DE L'HÉBERGEMENT}

Dans cette deuxième partie, nous commencerons par faire une présentation de la méthodologie utilisée et des particularités des petites structures étudiées (2.1). Nous aborderons ensuite le rôle d'Internet dans une logique de servuction en montrant que les petites structures étudiées utilisent leur site Internet non seulement dans une optique commerciale et marketing (2.2), mais également de renforcement et dévolution du positionnement concurrentiel, notamment quand le dirigeant présente certaines caractéristiques (2.3).

\subsection{Choix de la méthodologie qualitative et description des cas étudiés}

Nous avons volontairement focalisé notre attention sur les petites structures disposant d'un site Internet afin dêtre assurés d'une utilisation significative des TIC par nos entreprises, mais 
comme nous le montre l'annexe 1 (statistiques d'Eurostat), la présence d'un site Internet n'est pas garant d'une utilisation experte. Ainsi en France, si $50 \%$ des PE ont un site Internet, seules $12 \%$ d’entre elles l'utilisent pour la gestion des réservations (ce qui correspond à la catégorie la plus avancée des typologies mentionnées en partie 1). À titre de comparaison, c'est moins que l'Estonie, la Lituanie, la Slovaquie ou encore la Slovénie... Nous avons donc étudié, pendant lété 2010, quatre petites entreprises (moins de 50 salariés) de l'hébergement touristique dans les départements de la Charente-Maritime (région Poitou-Charentes) et de la Haute-Savoie (région Rhône-Alpes).

Notre question de recherche est caractérisée à la fois par la description et l'interprétation. Nous avons donc opté pour une méthodologie qualitative ; celle-ci est particulièrement pertinente lorsque l'objectif de recherche est d'explorer, de comprendre ou de générer des idées sur le pourquoi et le comment ${ }^{7}$. Afin d'apporter des éléments de réponse à notre problématique de recherche (sous quelles conditions la mise en place d'un site Internet peut-elle modifier le positionnement stratégique de petites structures d'hébergement dans le secteur du tourisme ?), nous avons établi, à partir de la littérature de la première partie, une grille d’analyse avec plusieurs questions clés.

\section{TABLEAU 1 : GRILle D’ANALYSE UTILISÉE POUR NOS ÉTUdES DE CAS}

1 Le site Internet permet-il d'accroitre la notoriété des petites structures et délargir la base de clients?

2 Le site Internet permet-il de mettre en œuvre de nouvelles techniques commerciales?

3 Le site Internet permet-il de fidéliser et d’entretenir la base de clients?

4 Le site Internet nécessite-t-il un alignement/une modification de l'organisation?

5 Le site Internet permet-il d’engager une réflexion sur la création de valeur et d'améliorer le positionnement concurrentiel ?

6 Le dirigeant joue-t-il un rôle majeur dans la création et l'utilisation du site Internet, notamment en raison de son profil et de son réseau?

Cette grille d'analyse a été utilisée pour conduire les entretiens semi-directifs avec nos dirigeants de petites structures. Nous avons parfois reformulé les questions à plusieurs reprises lors des entretiens afin de vérifier la cohérence des réponses apportées.

$7 \quad$ Nous utilisons les méthodologies décrites par Yin (1989) et Miles et Huberman (1994) : les données recueillies ont été organisées selon les techniques préconisées par Miles et Huberman (1994) en effectuant un travail de condensation des données, de présentation, délaboration et de vérifications des conclusions à chaque étape d'analyse. Notre codage est essentiellement un « codage thématique » à priori défini par Miles et Huberman (1994). 
Les entretiens semi-directifs d'une durée moyenne de deux heures avec les dirigeants avaient pour objet de comprendre les principales utilisations, les freins et les impacts des TIC en général et d'Internet en particulier, au sein de ces petites structures. Ainsi, lors des entretiens nous commencions par cerner l'identité du dirigeant (sa formation, son expérience) et celle de son entreprise (taille, date de création, métier, etc.). Ensuite, nous abordions l'utilisation d'Internet et les incitations qui ont poussé l'entreprise à séquiper (type de TIC utilisé, stratégie d'implantation, moyens mis en œuvre, etc.). Enfin, nous terminions l'entretien en essayant de cerner l'impact de la mise en place d'un site Internet sur les activités de l’entreprise et les bénéfices perçus. Nous nous sommes attachés à bien comprendre les motivations du dirigeant par rapport aux spécificités du secteur du tourisme qui est cyclique et soumis à une très forte concurrence, parfois avec des acteurs de taille beaucoup plus importante.

Nous avons systématiquement croisé les données des entretiens pour identifier les inférences par rapport à nos questions et lorsque nous avions un doute nous avons recontacté les dirigeants pour approfondir certains points. Les demandes de clarification ont été réalisées par courrier électronique ou par téléphone.

\section{ENCADRÉ 2 : COMPLÉMENTS D'INFORMATION ET DONNÉES SECONDAIRES}

Ces interviews ont été complétées par des données secondaires internes (notes internes, site Internet, etc.) et externes (extraits de presse). Nous avons croisé les propos des dirigeants avec les fonctionnalités des sites Internet afin d'éviter trop de subjectivité, ainsi qu'avec les plaquettes commerciales et les discours des prescripteurs. Nous avons ainsi réalisé six entretiens avec des prescripteurs ou organismes publics et privés : le conseiller tourisme de la CCI de La Rochelle, le responsable commercial du groupement Hôtels et Chalets de Tradition, le directeur commercial de RESO France et trois membres de RESO 7374. Ces acteurs nous ont permis d'avoir une vision plus large et de mieux cerner l'importance de l'environnement des petites structures en complétant les discours des dirigeants. Nous avons pu ainsi mieux comprendre les caractéristiques et les particularités des secteurs d’activités appartenant au tourisme. Les prescripteurs et les différents organismes rencontrés ont également facilité notre mise en relation avec les dirigeants de petites structures.

Chaque petite structure rencontrée dispose d'un site Internet et également d'autres applications (logiciels de gestion, CRM, etc.), le tout constituant le SI de l'entreprise même si ce dernier n'est pas forcément très élaboré de prime abord. Le site Internet représente loutil le plus important pour tous les dirigeants rencontrés. Nous avons donc focalisé notre attention sur le site Internet de la PE et ses fonctionnalités. 
ENCADRÉ 3 : Les 3 Îles (Île de RÉ, ThalassothéRAPIE) WWW.Thalasso-LaRochellesud.Fr/

Entreprise : 50 salariés, création en 1990, autonomie depuis 2008.

Activité : Soins + hébergement + restauration, clientèle européenne.

Répondant : Directeur et responsable commercial (46 ans), IUT génie mécanique et ESC.

TIC utilisées : Informations pratiques (encart promotionnel), visites virtuelles à $360^{\circ}$, webcam en temps réel, widget météo locale, vente de bons cadeaux en ligne... logiciel de gestion de la thalassothérapie, blog (en projet).

Encadré 4 : Les Cormorans (La Rochelle, Camping) wWw.Cormoran.com/

Entreprise : 3 à 10 pers. selon saison, création en 2002, affaire familiale.

Activité : Hébergement, clientèle européenne.

Répondant : Directeur (41 ans), DUT/expérience liée à la gestion du camping.

TIC utilisées : Site Internet traduit en six langues, photos, vidéos, infos diverses, possibilité de réserver en ligne directement, livre d’or... logiciel de gestion du camping, GED (en projet).

Encadré 5 : Hôtel ${ }^{* * *}$ Le Dahu (Morzine, Hôtellerie/ Restauration) www.dahu.com/

Entreprise : 8 à 36 pers. selon saison, création en 1955, affaire familiale reprise en 1986.

Activité : Hébergement + restauration + activités loisirs (moto, ski...), $50 \%$ clientèle internationale.

Répondant : Directeur (49 ans), formation hôtelière/cuisinier.

TIC utilisées: Site Internet traduit en anglais, réservations en ligne, lettre d'information, webcam, actualités diverses de l'hôtel... Intranet avec mot de passe pour les clients réguliers, réservation avec ReservIT, progiciel WinHôtel (CEGID).

Encadré 6 : Hôtel ${ }^{* * *}$ Le Macchi (Châtel, Hôtellerie / Restauration) WWW.HOTELMACCHI.COM/

Entreprise : 6 à 36 pers. selon saison, création en 1970, affaire familiale reprise en 1985.

Activités : Hébergement + restauration + activités loisirs (randonnées, ski, gym...), $40 \%$ clientèle européenne.

Répondant : Directeur et chef cuisinier (48 ans), formation hôtelière/cuisinier.

TIC utilisées : Site Internet traduit en cinq langues, possibilité de réserver en ligne, webcam, offres spéciales et forfaits, commandes de bons cadeaux... CRM pour envoyer des lettres d'information ciblées, progiciels de réservation : PMS winHôtel et WinRestau (de CEGID). 
Afin de rendre plus synthétique l'analyse de l'utilisation du site Internet par les petites structures étudiées, nous présentons nos résultats dans des tableaux qui reprennent les items de notre grille d'analyse. Nous commentons ensuite ces tableaux en illustrant chacune des dimensions (tableau 2 : dimensions marketing et commerciale, tableau 3 : dimension stratégique).

\subsection{La confirmation de l'utilisation d'Internet dans une logique de servuction}

Le premier constat est que nos cas, qui rappelons le, sont des entreprises à un stade avancé de l'utilisation d'Internet, utilisent de manière importante les fonctionnalités de leur site Internet pour développer les dimensions commerciales et marketing conformément à notre revue de littérature. Les trois premières questions de notre grille d’entretien nous ont permis de vérifier cela même si l’on constate quelques disparités entre les petites structures.

TABLEAU 2 : DÉVELOPPEMENT DE LA DIMENSION COMMERCIALE ET MARKETING VIA LES SITES INTERNET

\begin{tabular}{llcccc}
\cline { 3 - 5 } & $\begin{array}{c}\text { Les } \\
\text { Îlles }\end{array}$ & $\begin{array}{c}\text { Les } \\
\text { Cormorans }\end{array}$ & $\begin{array}{c}\text { Hôtel } \\
\text { Le Dahu }\end{array}$ & $\begin{array}{c}\text { Hôtel } \\
\text { Le Macchi }\end{array}$ \\
\hline Q1 & $\begin{array}{l}\text { Le site Internet permet-il } \\
\text { d'accroître la notoriété des } \\
\text { petites structures et délargir } \\
\text { la base de clients ? }\end{array}$ & ++ & +++ & +++ & +++ \\
\hline Q2 & $\begin{array}{l}\text { Le site Internet permet- } \\
\text { il de mettre en oeuvre } \\
\text { de nouvelles techniques } \\
\text { commerciales? }\end{array}$ & ++ & + & ++ & + \\
\hline Q3 & $\begin{array}{l}\text { Le site Internet permet-il de } \\
\text { fidéliser et dentretenir } \\
\text { la base de clients? }\end{array}$ & ++ & ++ & +++ & ++ \\
\hline
\end{tabular}

$(-)$ pas constaté, $(+)$ faiblement constaté, $(++)$ moyennement constaté, $(+++)$ fortement constaté

Laugmentation de la notoriété et de la base de clients grâce au site Internet (Q1) est mentionnée par tous les dirigeants. Ainsi, la motivation principale de l'utilisation d'un site Internet a été la possibilité pour ces petites structures délargir leur base clients en France comme à létranger. Très vite, au-delà de la notoriété que pouvait leur procurer une présence interactive et bien maitrisée sur Internet, les dirigeants se sont rendus compte de létendue des possibilités que pouvaient leur fournir conjointement un progiciel et un site Internet dynamique, notamment lorsque ce dernier est traduit en plusieurs langues. Par exemple, le dirigeant de l'hôtel Le Macchi a récemment fait traduire son site en russe pour exploiter cette clientèle (qui a ponctuellement commencé à fréquenter son hôtel) et renforcer sa notoriété auprès de ce pays. Le dirigeant du camping les Cormorans sur l'île de Ré indique que son site Internet traduit en six langues, génère $30 \%$ de ventes directes et plus de $50 \%$ des contacts clients (certains finalisent la transaction autrement que par le site). Il considère également que la proportion de clients 
étrangers est plus importante. Enfin, le centre de thalassothérapie les 3 Îles, réalise $25 \%$ de son chiffre d'affaires via son site Internet (achat de prestations, de produits et de bons cadeaux).

En revanche, si l'utilisation du site Internet des petites structures va dans le sens de la mise en place de nouvelles techniques commerciales (Q2), ces dernières ne sont pas agressives : le site ne sert pas vraiment à lisser l'activité, car même si nos entreprises connaissent deux pics saisonniers en hiver et été, les dirigeants ne veulent pas mettre en place des techniques commerciales qui "braderaient " leur service. On peut citer l'exemple du camping Les Cormorans dont le site Internet permet de faire des offres promotionnelles avec des bandeaux fonctionnant très bien pour les offres de dernières minutes qui permettent de s'ajuster à la concurrence et aux capacités de réservation. La stratégie n'est pas une stratégie de prix et, même si les statistiques de taux d'occupation fournies par l'outil informatique pouvaient tenter les responsables de pratiquer des prix cassés pour faire du remplissage en saison creuse, les petites structures ne veulent pas s'y résoudre. Le yield management est donc utilisé avec prudence, car les maîtres mots sont « qualité, service, satisfaction » et les dirigeants cherchent à conserver cette image en priorité. Ce résultat va en partie à l'encontre des résultats de Dyerson et al. (2009) qui montraient que la motivation principale de leurs entreprises résidait plus dans la réduction des coûts que dans la création de valeur ajoutée.

Létude des cas conforte également l'impact de certaines fonctions du site Internet sur la fidélisation des clients (Q3). Nos petites structures vont donc plus loin que celles que Lucchetti et Sterlacchini (2004) avaient observées. Par exemple, pour personnaliser la relation avec ses clients, l'hôtel Le Dahu a créé un club privé où les clients peuvent échanger des photos, des idées, etc., à distance avec les autres clients, après leur séjour. Ce club relate également la vie de l'hôtel tout au long de l'année et maintient un lien relationnel. Le dirigeant de ce même hôtel a également construit une base de données clients (avec une fiche par client comportant une centaine de caractéristiques). Cette base, alimentée régulièrement et couplée à un CRM, lui permet de proposer des offres personnalisées, et donc de fidéliser sa clientèle (50\% des clients du Dahu reviennent régulièrement). Le dirigeant du camping Les Cormorans de lîle de Ré indique également que les clients habituels regardent régulièrement le site Internet pour se tenir informés des nouveautés (une lettre d'information à leur intention est en phase de réalisation). Ces différentes actions via le site Internet ont vocation à renforcer la fidélisation des clients et à augmenter le chiffre d'affaires des petites structures qui sont très sensibles à la moindre variation de leur activité économique et de leur trésorerie. Cela contribue également à maintenir un lien personnalisé qui s'explique par la relation de proximité entre les dirigeants et leurs clients. Certains clients des deux hôtels sont d’ailleurs considérés comme « des membres de la famille».

Toutefois, les petites structures de notre étude rencontrent des difficultés, déjà recensées dans la littérature (Montazemi, 2006) : tout d'abord, elles doivent absorber le coût des progiciels ou de la construction d'un site Internet dynamique et interactif incluant par exemple un système de gestion de contenu (CMS, etc.) et sont donc obligées détaler leurs investissements dans le temps. Concernant plus particulièrement le site Internet, le coût de fonctionnement et de référencement sur des moteurs de recherche s'avère très important ( $3 \mathrm{~K} €$ annuel de fonctionnement pour le site Internet du camping les Cormorans, $18 \mathrm{~K} €$ annuel pour référencer sur Google le site du centre de thalassothérapie des Îles). Néanmoins, ce coût est intégré pleinement dans la gestion budgétaire des établissements et est considéré comme une dépense nécessaire pour accroître l'activité commerciale. 


\subsection{Internet comme renforcement du positionnement concurrentiel de la PE, notamment via son dirigeant}

Les travaux de Morisson et al. (1999) ont montré que les avantages liés aux TIC et à Internet peuvent s'avérer bénéfiques d'un point de vue stratégique : réduction des coûts, stratégie de différenciation, de diversification, recherche de nouvelles sources de revenus, ou encore amélioration de la performance de l'organisation. Dans les petites structures étudiées de notre recherche, à partir d'un certain niveau d'expertise sur les dimensions commerciale et marketing, le site Internet peut conduire à un renforcement voire une évolution du positionnement concurrentiel. Le site contribue ainsi à renforcer la différenciation, mais aussi le différentiel de valeur perçu par le client (Aldebert, 2008). Lévolution du positionnement stratégique n’est pas forcément prévue au départ par les dirigeants, mais si les bénéfices perçus sont significatifs, ces derniers vont progressivement intégrer le site Internet dans leur réflexion et le considérer comme une composante de la stratégie, et plus seulement comme un simple canal de distribution. Ainsi, l'hôtel Macchi a progressivement ajouté des services pour anticiper les demandes de sa clientèle étrangère, clientèle qui est arrivée via Internet. Le site Internet a donc permis, par effet de ricochet, de faire évoluer le positionnement stratégique vers le haut de gamme. Les dirigeants vont donc avoir un rôle déterminant dans la prise de décision qui consiste à aligner leur organisation et à adopter un positionnement concurrentiel plus qualitatif. On peut citer aussi le cas du camping Les Cormorans. La création du site Internet a accompagné la montée en gamme du camping qui a progressé en proposant des services plus qualitatifs (connexion Internet pour les clients, facilités de réservation, nouveaux mobil-homes, etc). Pour le centre de thalassothérapie, la création du site Internet a permis de conforter l'établissement avec une meilleure visibilité par rapport à ses concurrents directs au nombre de six dans la région. Le responsable considère ainsi que le site Internet et ses fonctionnalités permettent une stratégie de différenciation surtout « avec une offre considérée comme statique ».

TABLEAU 3 : ÉVOLUTION DU POSITIONNEMENT STRATÉGIQUE

\begin{tabular}{llcccc}
\cline { 2 - 5 } & $\begin{array}{c}\text { Les } \\
\text { 3̂les }\end{array}$ & $\begin{array}{c}\text { Les } \\
\text { Cormorans }\end{array}$ & $\begin{array}{c}\text { Hôtel } \\
\text { Le Dahu }\end{array}$ & $\begin{array}{c}\text { Hôtel } \\
\text { Le Macchi }\end{array}$ \\
\hline Q4 & $\begin{array}{l}\text { Le site Internet nécessite-t-il } \\
\text { un alignement/une modifica- } \\
\text { tion de l'organisation? }\end{array}$ & + & ++ & +++ & ++ \\
\hline Q5 & $\begin{array}{l}\text { Le site Internet permet- } \\
\text { il dengager une réflexion } \\
\text { sur la création de valeur et } \\
\text { d'améliorer le positionnement } \\
\text { concurrentiel ? }\end{array}$ & ++ & +++ & ++ & ++ \\
\hline Q6 & $\begin{array}{l}\text { Le dirigeant joue-t-il un rôle } \\
\text { majeur dans la création et } \\
\text { l'utilisation du site Internet, } \\
\text { notamment en raison de son } \\
\text { profil et de son réseau? }\end{array}$ & +++ & +++ & +++ & +++ \\
\hline
\end{tabular}

$(-)$ pas constaté, $(+)$ faiblement constaté, $(++)$ moyennement constaté, $(+++)$ fortement constaté 
Nos observations nous montrent que l'utilisation des TIC en général permet d’améliorer la gestion des petites structures et nécessite une modification de leur organisation qui passe d'abord par le back office à partir des données issues des clients et des prospects. Toutes nos entreprises ont vu une amélioration de leur gestion à un degré plus ou moins fort via une synchronisation de leurs différentes bases de données internes et une consultation des données en temps réel, ce qui permet une meilleure gestion de leur établissement. Les données qui transitent via le site Internet peuvent être utilisées pour une gestion en back-office, plus administrative, conduisant dès lors à un alignement des composantes du SI et une meilleure gestion interne (Q4). Ce constat est flagrant dans les deux hôtels où les dirigeants ne regrettent pas le temps où il fallait, tous les soirs, «synchroniser " manuellement les données de chaque ordinateur... Ils constatent également un gain de temps et une augmentation de la fiabilité grâce à la réservation en ligne. Toutefois, cela nécessite un investissement plus important pour connecter les différents systèmes entre eux et peut amener un degré de complexité supplémentaire, accentuant les risques pour les dirigeants. Lapproche est donc souvent progressive dans lélectronisation des processus de back et de front office. Certaines entreprises, comme le camping des Cormorans, vont encore plus loin et commencent à interconnecter leurs systèmes afin de permettre une gestion à distance.

En conséquence, l'avantage concurrentiel procuré par l'utilisation d'un site Internet permet à nos petites structures de renforcer leur différenciation (en proposant de nouveaux services, en ciblant leurs offres commerciales) et en créant des liens plus proches, voire intimes avec leur client (Bellaaj, 2009). C’est donc une approche très pragmatique qui est privilégiée par les dirigeants et qui contribue souvent à un positionnement qualitatif et donc plus haut de gamme (Q5). Ce résultat va d’ailleurs dans le sens de Qureshil et al. (2009) et confirme l'idée qu'un site Internet devient une vitrine de l'entreprise, mais également un élément de positionnement stratégique à part entière. Notre recherche montre que les petites structures peuvent utiliser leur site Internet comme un outil de différenciation par rapport à la concurrence, notamment sur les dimensions liées à la relation client, mais pas uniquement.

La réussite quant à l'utilisation d'un site Internet repose également sur un choix très judicieux de la technologie par rapport au positionnement de l'entreprise et de la nature de ses activités. Le développement des fonctionnalités du site Internet doit également se faire à partir d'une véritable réflexion quant au métier (et/ou la mission) de l'entreprise, car cela conditionne les services et la perception des consommateurs et des prospects. Ainsi, l'hôtelier doit se demander s'il est hébergeur ou créateur d'émotions, etc. Les différents entretiens réalisés avec les dirigeants et les prescripteurs montrent aussi l'importance d'effectuer une veille et de faire évoluer les fonctionnalités et les services, compte tenu non seulement des changements dans les comportements de consommation, mais également dans léquipement progressif des concurrents (comme le montre la courbe d'adoption des innovations de Rogers, 2003). Par exemple, les deux petites structures de la Charente-Maritime ont pour projet de développer des applications de géolocalisation sur des téléphones en lien avec leur site Internet. Elles offriraient alors des services plutôt proposés par des structures de taille plus importante ou haut de gamme. Le dirigeant du camping Les Cormorans allant même jusquà imaginer une logique de complémentarité avec les autres commerces proches, permettant d’influencer la décision des prospects.

Concernant les dirigeants, ils ne sont pas forcément technophiles, en ce sens qu'ils n'achètent pas forcément la dernière technologie à la mode, etc. Ils ont tous la quarantaine ou plus et 
n’ont pas reçu de formation orientée sur l'informatique ou les technologies. En revanche, c'est leur aptitude à percevoir l'utilité des technologies, leur profil innovant et preneur de risques qui semblent se dégager (Q6). Les responsables rencontrés font, par exemple, une veille sur les sites Internet des concurrents directs (design, services, et également taux d'occupation). L'un de nos cas, le responsable du centre de thalassothérapie, utilisait Netvibes ${ }^{8}$ comme outil $^{2}$ de veille par rapport à son activité professionnelle. Ce constat va dans le sens de Mehrtens et al. (2001) : le dirigeant, malgré son manque de connaissances pour piloter la mise en place d'un progiciel ou animer un site Internet, va faire des efforts pour maitriser loutil en passant du temps pour comprendre le fonctionnement (cas de l'hôtel Le Dahu) ou en embauchant dans un premier temps un collaborateur possédant les compétences techniques nécessaires (cas de l'hôtel Macchi). Cela correspond à un alignement de l'organisation et des ressources sur les TIC.

Quant aux acteurs externes (fournisseurs, clients, etc.), ils ne peuvent pas être considérés comme des incitateurs indirects : c'est un peu comme si, pris un à un, ces acteurs n'avaient pas de réel poids, en revanche leur agrégation constitue un facteur de motivation pour utiliser les TIC. Les prescripteurs (CCI, groupements, clubs, etc.) sont vus comme des acteurs sur lesquels le dirigeant peut s'appuyer pour orienter ou plutôt conforter ses choix. Ainsi, le responsable commercial de HCT endosse plusieurs casquettes : « je suis à la fois l'initiateur, l'apporteur de solutions technologiques, le conseiller; mon rôle est dêtre un peu une personne-ressource qui permet aux directeurs d'hôtels de s'appuyer sur quelqu'un en cas de besoin; ma présence les rassure et leur donne confiance quand il s'agit de se lancer dans une nouvelle techno ». Son rôle de sensibilisation et de veille pour les TIC est très important pour les dirigeants de petites structures qui disposent de peu de temps et de moyens. De plus, les prescripteurs sont des acteurs importants dans la mise en relation des entreprises et des prestataires techniques qui disposent de solutions adaptées. Cependant, ce rôle proactif des prescripteurs nous renvoie à la question de la différenciation : ainsi pourrait-on se demander si à terme les prescripteurs n'annulent pas la différenciation des utilisateurs précoces et donc, leur avantage concurrentiel en voulant diffuser les TIC parmi toutes les entreprises. Néanmoins, les facteurs déclencheurs (fortement impulsés par les prescripteurs) sont intimement liés entre eux et convergent vers le dirigeant qui semble jouer finalement, un rôle central.

\section{CONCLUSION}

La mise en place et l'utilisation des TIC en général et d'Internet, en particulier chez les PE de l'hébergement touristique, leur permettent certes d’obtenir une meilleure visibilité auprès de leurs clients, mais également les incitent à mieux structurer leur offre (pour plus de lisibilité) et à létoffer pour répondre au mieux aux diverses attentes de leur client : un véritable cercle vertueux se met en place. Progressivement, cette amélioration sur les dimensions commerciale et marketing les incite à mener une réflexion plus globale au niveau stratégique et leur permet, in fine, de clarifier leur positionnement concurrentiel. Ceci est, certes, bénéfique face à la

8 Netvibes peut se définir comme un portail Web gratuit personnalisable par chaque utilisateur à partir de ses choix de flux RSS, API... 
concurrence, mais pourrait aussi leur permettre d’avoir des arguments supplémentaires face aux partenaires potentiels afin d’obtenir plus facilement des fonds et/ou des aides si nécessaire.

En termes d'implications managériales, notre recherche montre que :

- Internet peut être un moyen d'innover dans loffre de services pour contribuer à un positionnement de différenciation par le haut, voire de niche (personnalisation de l'offre, renforcement de la relation client, amélioration de la visibilité face à des concurrents de plus grande taille...);

- le site Internet devient la première représentation du positionnement de la petite structure lui permettant de rivaliser avec des entreprises de taille plus importante si elles sont au stade le plus avancé en matière de fonctionnalités ;

- il n'est pas nécessaire que le dirigeant soit un utilisateur précoce, ce qui importe, c'est sa sensibilité aux TIC et sa prise de conscience de l'importance de la mise en place des TIC. Les parties prenantes, partenaires ou encore le réseau vont jouer un rôle d'incitateur ou de "support " pour le dirigeant : cet entourage aide le dirigeant à surmonter les coûts (humains, financiers, etc.) liés à l'installation et la maintenance d'un site Internet en particulier et des TIC en général ;

- enfin, il faut mettre la technologie au service d'une stratégie, surtout pour les petites structures qui ont peu de moyens et qui nont pas de « droit à l'erreur » : les TIC seules n’ont pas de caractère à créer un avantage concurrentiel, mais c'est lorsquelles sont combinées à d'autres ressources qu’elles viennent soutenir la stratégie et créer de la valeur.

Naturellement, notre recherche comporte des limites, inhérentes à l'approche qualitative adoptée, comme le faible nombre d’entreprises interrogées qui ne nous permet pas de tirer de conclusions générales pour les PE. Néanmoins, cette limite est à nuancer, car les PE du tourisme (de Charente-Maritime et Haute-Savoie) avancées en termes d'utilisation des TIC sont peu nombreuses et difficiles à identifier à priori d’où notre difficulté à les repérer puis à obtenir leur accord pour un entretien. Enfin, le choix du secteur du tourisme peut être considéré comme une limite, car en avance dans l'utilisation des TIC.

Cette étude exploratoire ouvre plusieurs perspectives de recherche. Nous pourrions tout d'abord mener une étude quantitative sur un plus large échantillon représentatif de PE en lélargissant aux trois autres catégories de Daniel et al. (2002) pour tester empiriquement nos résultats. Ainsi, l'apport de données quantitatives pourrait-il nous permettre de mesurer la participation de l'utilisation des TIC au développement de lentreprise via la mise en place d'indicateurs classiques (CA, taille...). Enfin, il pourrait être pertinent de retravailler les typologies existantes pour voir s'il est nécessaire d'avoir d'autres catégories en lien avec les innovations liées aux TIC et à Internet (applications de géolocalisation, paiement sans contact, réseaux sociaux, etc.) afin de mieux identifier les choix stratégiques des acteurs, y compris ceux de petite taille. 
Annexe 1 : Utilisation de Sites Internet et de Pages Web pour les PE dans Les Pays EUROPÉENS EN 2009 (EN \%)

\begin{tabular}{|c|c|c|c|}
\hline Pays (UE) & $\begin{array}{l}\text { Entreprises } \\
\text { ayant un site } \\
\text { Internet ou } \\
\text { une page } \\
\text { d'accueil }\end{array}$ & $\begin{array}{c}\text { Entreprises } \\
\text { où le site Internet } \\
\text { propose } \\
\text { un catalogue de } \\
\text { produits ou une } \\
\text { liste de prix }\end{array}$ & $\begin{array}{c}\text { Entreprises } \\
\text { où le site Internet } \\
\text { permet } \\
\text { une commande ou } \\
\text { réservation } \\
\text { en ligne }\end{array}$ \\
\hline Belgium & 74 & $:$ & $:$ \\
\hline Bulgaria & 29 & 24 & 7 \\
\hline Czech Republic & 68 & 40 & 24 \\
\hline Denmark & 86 & 45 & 22 \\
\hline Germany & 76 & 35 & 22 \\
\hline Estonia & 64 & 37 & 12 \\
\hline Ireland & 57 & 33 & 16 \\
\hline Greece & 58 & $:$ & $:$ \\
\hline Spain & 52 & 29 & 6 \\
\hline France & 50 & 32 & 12 \\
\hline Italy & 56 & 35 & 14 \\
\hline Cyprus & 43 & 36 & 6 \\
\hline Latvia & 37 & 25 & 7 \\
\hline Lithuania & 56 & 35 & 13 \\
\hline Luxembourg (Grand-Duché) & 64 & 32 & 12 \\
\hline Hungary & 47 & 42 & 13 \\
\hline Malta & 60 & 43 & 16 \\
\hline Netherlands & 82 & 22 & 22 \\
\hline Austria & 77 & 46 & 16 \\
\hline Poland & 52 & 38 & 6 \\
\hline Portugal & 43 & 24 & 9 \\
\hline Romania & 23 & 15 & 7 \\
\hline Slovenia & 64 & 57 & 14 \\
\hline Slovakia & 68 & 45 & 17 \\
\hline Finland & 82 & 30 & 12 \\
\hline Sweden & 85 & $:$ & $:$ \\
\hline United Kingdom & 72 & $:$ & $:$ \\
\hline Croatia & 52 & 29 & 6 \\
\hline
\end{tabular}

Source : Eurostat 2010 ( : données non disponibles)

\section{BIBLIOGRAPHIE}

ACAR E., KACAK I., SEY Y. et ARDITI D. (2005), Use of information and communication technologies by small and mediun-sized enterprises (SMEs) in building construction, Construction Management and Economics, 23, 713-722. 
ALDEBERT B. (2008), Technologies de l'information et de la communication : un outil stratégique pour les petites et moyennes entreprises touristiques, Management et Sciences Sociales, 5, 11-30.

ALDEBERT B. et GUEGUEN G. (2009), Intérêt du dirigeant de PME touristiques pour les TIC : quels effets sur l'utilisation de la performance ?, $14^{e}$ colloque de l'AIM.

ALDEBERT B. et LOUFRANI-FEDIDA S. (2010), Repérer les compétences pour mieux comprendre le processus d'innovation. Le cas d'une TPE touristique, Revue Internationale P.M.E, 23, nº 1, 33-61.

AMABILLE S. et GADILLE M. (2006), Les usages d'Internet, facteurs de compétitivité de la « PME réseau »?, Finance Contrôle Stratégie, 9, 1, 35-57.

AMABILE S. et GADILLE M. (2003), Les NTIC dans les PME : Stratégies, capacités organisationnelles et avantages concurrentiels, Revue Française de Gestion, 29, 144, 43-63.

BECKINSALE M., LEVY M. et POWELL P. (2006), Exploring Internet Adoption Drivers in SMEs, Electronic Markets, 16, 4, 361-370.

BELLAAJ M. (2009), Effets modérateurs des capacités complémentaires dans le e-commerce. Application au contexte des PME, Revue Internationale P.M.E, 22, 2, 9-35.

BERGERON F., RAYMOND L. et CROTEAU A.M. (2001), Comportements stratégiques, choix et gestion des systèmes d'information : contribution à la performance, SIM, 4, 6, 5-26.

BONN M.A., FURR H.L. et SUSSKIND A.M. (1998), Using the Internet as a Pleasure Travel Planning Tool: an Examination of the Sociodemographic and Behavioral Characteristics Among Internet Users and Nonusers, Journal of Hospitality \& Tourism Research, 22, 303-317.

BURGESS S. (2002), Managing Information Technology in small business: Challenges and solutions, Idea Group Publishing.

CALDEIRA M.M. et WARD J.M. (2002), Understanding the successful adoption and use of IS/IT in SMEs: an explanation from Portuguese manufacturing industries, Information Systems Journal, 12, 2, 121-152.

CRAGG P., KING M. et HUSSINC H. (2002), IT alignment and firm performance in small manufacturing firms, The Journal of Strategic Information Systems, 11, 2, 109-132.

DANIEL, E., WILSON, H. et MYERS A. (2002), Adoption of e-commerce by SMEs in the UK, towards a stage model, International Small Business Journal, 20, 3, 253-270.

DYERSON, R., HARINDRANATH G. et BARNES D. (2009), National Survey of SMEs' Use of IT in Four Sectors, The Electronic Journal Information Systems Evaluation, 12, 1, 39-50.

FRANCE NUMÉRIQUE 2012 (2008), Plan de développement de léconomie numérique, rapport du secrétariat d'état chargé de la prospective, de l'évaluation des politiques publiques et du développement de léconomie numérique, $81 \mathrm{p}$.

GENSOLLEN M. (2001), Internet : marché électronique ou réseaux commerciaux ?, Revue Économique, $52,6,133-158$.

HARINDRANATH G., DYERSON R. et BARNES D. (2008), ICT Adoption and Use in UK SMEs: A Failure of Initiatives?, The Electronic Journal Information Systems Evaluation, 11, 2, 91-96.

HENDERSON J.C. et VENKATRAMAN N. (1993), Strategic Alignment: leveraging Information Technology for Transforming Organizations, IBM Systems Journal, 32, 1, 4-16.

JULIEN P.A. (1998), Les PME, bilan et perspectives, Édition Economica, Paris, $2^{\mathrm{e}}$ éd., 351 p.

JULIEN P.A. et MARCHESNAY M. (1988), La petite entreprise, Vuibert.

KALIKA M. et RIVAL Y. (2009), Évaluer la performance de l'entreprise induite par l'usage de portails Web - le cas du secteur touristique, SIM, 14(1), 75-136. 
LEE, J. et A.M. MORRISON (2010), A comparative study of web site performance, Journal of Hospitality and Tourism Technology, 1, 1, 50-67.

LEVENBURG N.M. (2005), Does Size Matter? Small Firms' Use of E-Business Tools in the Supply Chain, Electronic Markets, 15, 2, 94-105.

LUCCHETTI R. et STERLACCHINI A. (2004), The Adoption of ICT among SMEs: Evidence from an Italian Survey, Small Business Economics, 23, 151-168.

MARCHESNAY M. (2003), La petite entreprise : sortir de l'ignorance, Revue Française de Gestion, 144, 107-118.

MATTHEWS P. (2007), ICT Assimitation and SME Expansion, Journal of international Development, 19, 817-827.

MCDONAGH P. et PROTHERO A. (2000), Euroclicking and the Irish SME: Prepared for ecommerce and the single currency, Irish Marketing Review, 13(1), 21-33.

MEHRTENS J., CRAGG P.B. et MILLS A.M. (2001), A model of Internet adoption by SMEs, Information and Management, 39 (3), 165-176.

MILES M.B. et HUBERMAN A.M. (1994), Qualitative Data Analysis: An expanded sourcebook (2éd.), Sage :London \& Thousand Oaks, California.

MONNOYER-LONGÉ M.-C. (2003), PME et technologies de l'information. De la prise de décision à la mise en œuvre, Revue Internationale P.M.E, 15, 3-4, 11-36.

MONNOYER-LONGÉ M.-C. et MADRID C. (2007), Intégrer les sites web dans les stratégies, Revue Française de Gestion, 4, 173, 145-155.

MONTAZEMI A.R. (2006), How They Manage IT: SMES in Canada and the U.S, Communication of the $A C M, 49,12,109-112$.

MORRISON A.M., TAYLOR S. , MORRISON A.J. et MORRISON A.D. (1999), Marketing small hotels on the World Wide Web, Journal of Information Technology \& Tourism, 2, 97-113.

POLLARD C.E. et HAYNE S.C. (1998), The changing faces of information systems issues in small firms, International Small Business Journal, avril-juin, 16(3), 70-87.

QIAN C.Z, CLARKE G.R et HALEWOOD N. (2006), The Role of ICT, Doing Business Information and Communications for Development - Global Trends and Policies, World Bank : Washington DC.

QURESHIL S., KAMAL M. et WOLCOTT P. (2009), Information Technology Interventions for Growth and Competitiveness in Micro-Entreprise, International Journal of E-Business Research, 5, 1, 117-140.

RAYMOND L., BERGERON F. et BLILI S. (2005), The assimilation of E-business in manufacturing SMEs: determinants and effects on growth and internationalization, Electronic Markets, 15(2), 106-118.

REIX R. (2002), Systèmes d'Information et management des organisations, éd. Gestion, Paris.

ROGERS E.M. (2003), Diffusion of innovations, $5^{e}$ éd., New York, Free Press.

SCAGLIONE M., SCHEGG R. et MURPHY J. (2009), Website adoption and sales performance in Valais' hospitality industry, Technovation, 29, 625-631

SULLIVAN B.C. (1985), Economics of Information Technology, International Journal of Social Economics, Bradford, 12, 1, 37-53.

YIN R. (1989), Case study research: Design and methods (Rev. ed.), Newbury Park, CA: Sage Publishing. 